\section{Breaking the silence}

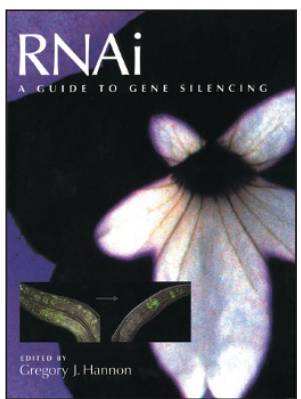

\section{RNAi: A Guide to Gene Silencing}

Edited by Gregory J Hannon

Cold Spring Harbor Press, 2003

436 pp. hardcover, $\$ 130.00$

ISBN 0-87969-641-9

\section{Reviewed by Gary Ruvkun}

When a field has exploded like the microRNA and RNAi field has, it is useful for those at the center of the action, as well as the multitudes affected by the sea change, to have an intellectual framework to which to turn. Somehow, the reviews published in journals never seem to capture how we got to where we are now; they are so focused on the most recent hot news that they are rarely synthetic. And in a competitive field, it is difficult to convince the leaders to slow down enough to think deeply or reveal their best new ideas. So I was worried that a full book on RNAi would not work, that the field is too hot and moves too fast for any book to be useful for more than a few months after it is published and that the best people in the field would not take the time to write.

RNAi: A Guide to Gene Silencing is a compendium, with many chapters written by leaders in the field. Most interesting to me were the chapters on the early days of post-transcriptional and transcriptional gene silencing (PTGS and TGS, respectively) in plants, written by Richard Jorgenson and by Antonius Matzke and colleagues. It is inspirational to see how talented molecular biologists, studying problems of plant transgenesis and engineering, were drawn by their observations into the RNAi field. It is a tale of science away from the self-anointed centers of molecular biology (read: animal biology), at a now-departed biotechnology company in Oakland, California, or at

Gary Ruvkun is in the Department of Molecular Biology, Wellman 8, Massachusetts General Hospital, Department of Genetics, Harvard Medical School, Boston, Massachusetts 02114, USA.

e-mail:ruvkun@molbio.mgh.harvard.edu plant molecular biology institutes in Versailles, Salzburg and Norwich. It is a tale of the plant molecular biology community scooping the rest of biology by studying more diversity, like the very strange viroids of plants, tiny circles of RNA that led to the discovery of TGS and PTGS. It was a group that first used surrogate genetic techniques, anti-PTGS proteins (again from rather obscure viruses), to dissect the steps of the process. In a nutshell, it is heroic science. And because the work was published, in many cases, in specialized plant journals, or written for the plant audience even when published in journals with a more general readership, it is useful that the work is presented here in some historical context and using the new language of RNAi, a language now understood by many. These chapters alone are worth the price of the book.

Also not generally available in reviews on RNAi are details of RNAse III enzymology and how it is involved in ribosome biogenesis, as provided in the chapter by Allen Nicholson. This chapter is an important addition to the book as RNase III is relevant to the study of the Dicer ribonuclease, a key component of the RNAi machinery.

But there are some weaknesses to the book. Hannon can be commended for recruiting many excellent contributors, but he is a better scientist than book editor. For instance, reading the entire canon of lin-4, Dicer and double-stranded RNA in nearly every chapter was tiresome. In this sense, the book is like a compendium of stand-alone review articles on related themes that do not form a well-integrated whole. In addition, the book navigates a strange path between classic review-style chapters and methods sections with recipes. The cookbook protocols may be useful to some, but given that this is really not a methods book and most of the methods chapters almost seem to be lifted from introductions to grant applications, I worry about the care put into the protocols.

Sorely missing from any book aspiring to be the definitive monograph on RNAi are chapters from some of the leading figures in the field (David Baulcombe, Andrew Fire, Craig Mello and Victor Ambros are absent). They probably could not be persuaded to contribute to a book when there are so many opportunities in high-visibility journals to write about the field. Then again, they may opt for a different forum in which to report on their early work. In the meantime, Greg Hannon's compendium stands out as the most comprehensive review of the RNAi field to date and thus serves as a useful reference for the growing number of scientists touched by this burgeoning field. 